\title{
General practitioners' attitudes on who should manage metabolic dysregulations associated with antipsychotics
}

\author{
Emma Bainbridge, ${ }^{1}$ Anne Gallagher, ${ }^{1}$ Gary McDonald, ${ }^{2}$ Colm McDonald, ${ }^{2}$ Mohamed Ahmed ${ }^{2}$
}

The Psychiatrist (2011), 35, 213-215, doi: 10.1192/pb.bp.110.031351

${ }^{1}$ Department of Psychiatry, University Hospital, Galway, Ireland; ${ }^{2}$ Department of Psychiatry, National University of Ireland, Galway, Ireland

Correspondence to Mohamed Ahmed (mohamed.ahmed@nuigalway.ie)

First received 1 Jun 2010, final revision 14 Oct 2010, accepted 23 Nov 2010
Aims and method To assess attitudes of general practitioners (GPs) on who should be responsible for managing metabolic dysregulations associated with the use of antipsychotics prescribed by psychiatrists. A total of 121 GPs in West Galway catchment area were surveyed using a simple questionnaire.

Results A total of $104 \mathrm{GPs}$ responded ( $86 \%$ response rate). The vast majority of responders (82\%) believed that medical management should be provided within primary care. However, $46 \%$ indicated that initial screening and simple non-pharmacological management should be provided by mental health services.

Clinical implications The vast majority of GPs appear willing to take over the medical management of metabolic dysregulations emerging from antipsychotic prescribing in secondary care. Clearly defined roles for mental health services and primary care in the management of metabolic complications are of paramount importance, and individual mental health services should implement protocols for screening, non-pharmacological management and referral to primary care.

Declaration of interest None.
There is strong evidence that people with severe and enduring mental illness have increased morbidity and mortality compared with the general population. This may be due to a combination of risk factors, including poor diet, excessive smoking, underrecognition of medical risk factors and reduced access to care. ${ }^{1}$ In recent years, treatment with antipsychotic medications has been recognised as an additional risk factor for the development of metabolic abnormalities in this group of patients. ${ }^{2}$ These abnormalities include weight gain, hyperlipidaemias, glucose dysregulation and diabetes mellitus. ${ }^{2}$

There is evidence to suggest that people with mental health problems are less likely than the general population to seek medical help for their physical problems. Reasons suggested for this include apathy, mistrust of the medical profession, and the fact that a large proportion of those patients are not registered with a general practitioner (GP). ${ }^{3}$ Kendrick found that people with long-term mental illness tend to seek medical attention only in crisis situations. ${ }^{4}$

In spite of clear clinical guidelines to monitor people receiving atypical antipsychotic medications, the health needs of this group of patients are often not addressed adequately by clinicians in specialised mental health services or in primary care settings. ${ }^{5}$ This is likely to be contributed to by continuing controversy over who should be responsible for the monitoring and management of complications of the medications prescribed by psychiatrists. ${ }^{5}$
Psychiatrists may not consider themselves adequately experienced or trained to deal with metabolic complications, whereas GPs may be reluctant to accept the increased burden of care entailed and may believe that psychiatrists should upskill to manage the complications of the medications that they prescribe.

Kendrick et al surveyed GPs on who should screen for physical problems in people with mental illness. Overall, 77\% thought that GPs should do this, while a larger percentage (93\%) thought GPs should manage these physical problems. ${ }^{6}$ In Ireland, O’Brien et al suggested that the high prevalence of physical illness and physical risk factors in people with mental illness warrants primary care involvement in screening for and treatment of such physical risk factors. ${ }^{7}$

The UK National Institute for Health and Clinical Excellence (NICE) guidelines recommend that primary care providers should monitor and manage the physical health aspects of people with schizophrenia. ${ }^{8}$ The American Psychiatric Association and other North American guidelines recommend that mental healthcare providers should monitor and provide some treatment if any physical health abnormalities are identified, particularly when these abnormalities occur as an adverse result of prescribed psychiatric treatment. ${ }^{9,10}$ Following a comprehensive review of major guidelines, Cohn \& Sernyak concluded that monitoring of, but not necessarily medical treatment of, 
metabolic disorders falls within the scope of psychiatric practice. $^{5}$ The Royal College of Psychiatrists' position is that monitoring is left up to local agreements between primary care and secondary mental health services; the College recommends liaison when physical assessments and the required management fall beyond the prescriber's level of competence. $^{11}$

In this study, we surveyed all GPs providing primary healthcare in Galway West catchment area to ascertain their views on who should be responsible for managing metabolic abnormalities in patients prescribed antipsychotic medications, and the most appropriate management line when such abnormalities are detected.

\section{Method}

A total of 121 GPs were identified from the local primary healthcare lists and sent the study questionnaire together with a stamped addressed envelope. Reminders were sent after 3 months.

The questionnaire was designed by the authors and primarily asked what management action is most appropriate when lipid and glucose abnormalities are detected in patients prescribed antipsychotic medications. The GPs were asked to select one of the four following options and to provide any additional comments.

A Patient should be referred to primary care for management of any metabolic complications.

B Initial simple management efforts should be made by psychiatry services (e.g. advice on nutrition/exercise), with referral to primary care only if these measures fail.

C Metabolic complications should be managed entirely by the psychiatric services. If medications are indicated, they should be prescribed by a psychiatrist, with GP input only if such management is unsuccessful.

D Metabolic complications should be managed entirely by the psychiatric services and patients referred directly to endocrinology services if needed.

\section{Results}

The initial response rate was $62 \%(n=75)$; after reminders were sent out, the total response rate rose to $86 \%(n=104)$. The number and percentage of respondents in each category were as follows:
A $37(36 \%)$
B $48(46 \%)$
C $6(6 \%)$
D $9(9 \%)$
Undetermined 4 (3\%)

Comments from those who selected option A indicated that GPs felt that they are best placed to manage metabolic complications and refer patients to endocrinology if needed (Box 1). There was also concern about a potential reduction in primary care attendance if patients' medical care was taken over by psychiatrists, and that this would put further pressures on the resources available in mental health

\section{Box 1 Selected quotes from questionnaire responses}

Option A

'Not to manage such complications in primary care would lead to further dissociation of psychiatric patients from GP services.'

'If mental health services are consumed by this, there will be no capacity to assess and manage our psychiatric patients when they are ill.'

'I strongly feel this is a primary care issue and that GP practice is best equipped to follow up these patients.'

Option B

'A combined management approach between primary care and psychiatry would be most appropriate.'

'It is imperative that I am aware of abnormal results as I am best placed to look for and address other risk factors for cardiovascular disease.'

'Exercise programmes should be available to patients on antipsychotic medications and perhaps clinics should be set up alongside the psychiatry services, in tandem with out-patient appointments to address lifestyle and dietetic issues.'

Option C

'Psychiatrists should manage metabolic complications as they are prescribing the antipsychotics.'

'Mental health teams were not keen to manage even minor complications.'

Option D

'To avoid delays in treatment, mental health teams should manage and refer directly to endocrinology if needed.'

'Patients with mental illness do not regularly attend primary care and for this reason GPs are not best placed to manage their metabolic complications.'

services, with subsequent negative impact on other psychiatric referrals from primary care.

General practitioners selecting option B preferred a combined approach, with guidance from psychiatry on monitoring needs, according to the medications prescribed and the associated risks. They felt that they were in a better position to then explore and address other risk factors.

General practitioners selecting option C commented that, as prescribers, psychiatrists have responsibility for monitoring and managing complications.

General practitioners selecting option D commented that there should be direct specialist-to-specialist referral when an endocrinological or cardiovascular concern is raised, bypassing primary care.

\section{Discussion}

The study findings illustrate that the vast majority of GPs believe that the medical management of metabolic complications of prescribed antipsychotic medications should be retained in primary care. However, approximately half of the sample believed that initial simple management efforts, such as advice on diet and exercise, should be made by psychiatrists before referring back to primary care if such measures fail. 
A significant minority of GPs believe that metabolic complications should be managed entirely by mental health services. One concern voiced to support this position is the lack of regular contact between some patients and their primary care service. Similar concerns were reported by Bosworth et $a l^{12}$ and Nasrallah et al. ${ }^{10}$ The latter concluded that routine health monitoring for people with schizophrenia should be performed by psychiatrists, particularly when certain conditions may be adverse results of prescribed psychiatric treatment. ${ }^{10}$ Santhouse \& Holloway suggested that psychiatrists should always try to identify physical problems, even if they are not expected to treat all of these problems. This is because psychiatrists are likely to be the first to notice a change in a patient's physical condition. $^{3}$

Current antipsychotic treatment guidelines recommend regular monitoring of body mass index, fasting lipid and glucose levels, and blood pressure. ${ }^{13}$ There seems to be a general consensus that such monitoring falls within the scope of mental health services, ${ }^{5}$ and therefore those services must be equipped to perform regular physical health checks and offer advice on the management of modifiable medical risk factors such as diet and lifestyle. This also necessitates establishing effective means of communicating abnormal findings to primary care providers to ensure appropriate medical management and follow-up.

Mental health teams should ensure that all patients are registered with GPs, and patients should be encouraged to present for checks of their physical health. There is a role for the education of patients about a range of lifestyle issues such as diet, exercise and smoking. ${ }^{3}$

Mental health services and local primary care providers should agree on clear guidelines outlining responsibilities and protocols for screening for and managing these serious metabolic abnormalities in a shared and integrated model of healthcare provision. ${ }^{14}$ The authors of this paper implemented a screening protocol for metabolic profile for patients receiving clozapine antipsychotic therapy at the Department of Psychiatry, University Hospital, Galway. As part of this protocol, any abnormal findings have to be reported back to GPs to decide on management options.

The strengths of the study are the high response rate and the inclusion of GPs working in rural and urban settings with various levels of deprivation. The findings are likely to be generalisable to the wider population of GPs in Ireland. However, the structure and funding of service provision in different countries are likely to necessitate variable guidelines; in particular, services that financially incentivise GPs to monitor and manage metabolic complications are more likely to meet with a positive response to shifting this burden of care from secondary to primary care. ${ }^{15}$

In conclusion, this study highlights that the majority of GPs believe they should be responsible for the monitoring and management of metabolic complications of antipsychotic medications once simple identification and perhaps non-pharmacological interventions have been implemented by mental health services. It is incumbent on mental health services to implement protocols to identify and implement care pathways for people with these serious complications.

\section{About the authors}

Emma Bainbridge and Anne Gallagher are registrars in psychiatry at University Hospital, Galway, Ireland. Gary McDonald is a lecturer in psychiatry, and Colm McDonald is a professor of psychiatry, in the Department of Psychiatry, National University of Ireland, Galway, Ireland. Mohamed Ahmed is a consultant psychiatrist at Limerick Mental Health Services and Honorary Clinical Fellow at the Department of Psychiatry, National University of Ireland, Galway, Ireland.

\section{References}

1 Haag S, Lindblom Y, Mjorndal T, Adolfsson R. High prevalence of the metabolic syndrome among a Swedish cohort of patients with schizophrenia. Int Clin Psychopharmacol 2006; 21: 93-8.

2 Nasrallah HA, Newcomer JW. Atypical antipsychotics and metabolic dysregulation: evaluating the risk/benefit equation and improving the standard of care. J Clin Psychopharmacol 2004; 24 (suppl 1): S7-14.

3 Santhouse A, Holloway F. Physical health of patients in continuing care. Adv Psychiatr Treat 1999; 5: 455-62.

4 Kendrick T. Management of people with schizophrenia in primary care. Adv Psychiatr Treat 1998; 4: 46-52.

5 Cohn TA, Sernyak MJ. Metabolic monitoring for patients treated with antipsychotic medications. Can J Psychiatry 2006; 51: 492-501.

6 Kendrick T, Sibbald B, Burns T, Freeling P. Role of general practitioners in care of long term mentally ill patients. BMJ 1991; 302: 508-10.

7 O'Brien SM, Devitt E, Ahmed M, McDonald C. High prevalence of risk factors for physical illness in a long-stay psychiatric unit. Ir J Psychol Med 2007; 24: 55-8.

8 National Institute for Health and Clinical Excellence. Schizophrenia: Core Interventions in the Treatment and Management of Schizophrenia in Adults in Primary and Secondary Care. NICE, 2002 [updated March 2009] (http://www.nccmh.org.uk/guidelines_schiz_update.html).

9 American Diabetes Association, American Psychiatric Association American Association of Clinical Endocrinologists, North American Association for the Study of Obesity. Consensus development conference on antipsychotic drugs and obesity and diabetes. Diabet Care 2004; 27: 596-601.

10 Nasrallah HA, Meyer JM, Goff DC, McEvoy JP, Davis SM, Stroup TS et al. Low rates of treatment for hypertension, dyslipidemia, and diabetes in schizophrenia: data from the CATIE schizophrenia trial sample at baseline. Schizophr Res 2006; 86: 15-22.

11 Royal College of Psychiatrists. Physical Health in Mental Health: Final Report of a Scoping Group. Royal College of Psychiatrists, 2009 (http:// www.rcpsych.ac.uk/files/pdfversion/OP67.pdf).

12 Bosworth $\mathrm{HB}$, Calhoun PS, Stechuchak KM, Butterfield MI Use of psychiatric and medical health care by veterans with severe mental illness. Psychiatr Serv 2004; 55: 708-10.

13 De Hert M, Van Eyeck D, De Nayer A. Metabolic abnormalities associated with second generation antipsychotics: fact or fiction? Development of guidelines for screening and monitoring. Int Clin Psychopharmacol 2006; 21: 1-5.

14 Barnes TR, Paton C, Cavanagh MR, Hancock E, Taylor DM. A UK audit of screening for the metabolic side effects of antipsychotics in community patients. Schizophr Bull 2007; 33: 1397-403.

15 Burns T, Cohen A. Item-of-service payments for general practitione care of severely mentally ill persons: does the money matter? $\mathrm{Br} J \mathrm{Gen}$ Pract 1998; 48: 1415-6. 